\title{
USO DE LAS TÉCNICAS DE EVALUACIÓN MULTICRITERIO EN SISTEMAS DE COSECHA FORESTAL DE MÍNIMO IMPACTO EN EL SUELO
}

\begin{abstract}
Oscar Bustos Letelier ${ }^{1}$, Carlos Mena Frau ${ }^{2}$
RESUMEN

El presente estudio tiene como objetivo la aplicación de una metodología para la selección de sistemas de cosecha forestal, de acuerdo a criterios económicos, productivos y ambientales, con el fin de identificar un sistema que cause el menor daño sobre el suelo forestal. Para ello se efectuó por un lado, una revisión bibliográfica detallada de los estudios realizados en la temática y por otro evaluaciones en terreno, con el objeto de identificar las variables del suelo que pudieran ser afectadas, como también de las características de las maquinarias que estuvieran relacionadas con las alteraciones del suelo. Una vez identificadas tales variables, se estableció una base de datos, la cual fue integrada mediante la aplicación de la técnica de evaluación multicriterio.
\end{abstract}

De acuerdo con lo anterior, se compararon las características de los distintos modelos de maquinaria involucrados en las faenas de cosecha forestal, observando que ninguno de ellos fuera superior al resto en todos los criterios utilizados para su evaluación. Cualquier alternativa fue superior al resto en por lo menos un criterio. Por esto la selección de una alternativa adecuada dependió de los objetivos que presentó el ente decisor, ya que una alternativa seleccionada no fue superior al resto en todas las condiciones de trabajo posibles, sólo en aquella en que se realizó el estudio.

Los modelos de maquinaria seleccionados que presentaron mejores características de trabajo y un menor daño al suelo, fueron: Grapple Skidder Caterpillar 528, Feller Buncher Timberjack 2628, y Forwarder Timberjack 1210B.

Palabras clave: Maquinaria forestal, ambiente forestal, técnicas de evaluación multicriterio.

1-Ingeniero Forestal, M.Sc. Facultad de Ciencias Forestales, Universidad de Talca, Chile, obustos@utalca.cl -

2-Cartógrafo, Dr. en Geomática, Facultad de Ciencias Forestales, Universidad de Talca, Chile, cmena@utalca.cl 


\section{ABSTRACT}

A methodology for selection of the harvest systems, according on economic, productive and environmental approaches was evaluated. The objective was to select those harvest systems that produces the lowest damage on forest soil. From several studies and ground evaluations, the main variables of soils and machineries that caused damage were identified. The integration of this data base was made by a multicriteria evaluation technique.

A comparison of different machineries models was performed, considering as premise that any of them was superior to the rest in all the approaches used for their evaluation. In this way any alternative was superior to the rest in at least one criterion. Therefore, the selection of an appropriate alternative will depend on the objectives that possess the expert.

Within the forest harvest systems models evaluated, those that presented better productive characteristics and minor damage to soil were Grapple Skidder Caterpillar 528, Feller Buncher Timberjack 2618 and Forwarder Timberjack 1210B.

Keywords: Forest machinery, forest environment, Multicriteria Evaluation Techniques. 


\section{INTRODUCCIÓN}

La extracción de la madera desde zonas boscosas requiere de la utilización de maquinaria de cosecha, la cual se caracteriza por una alta inversión de capital, como por las exigencias de una alta efectividad y eficiencia, tanto en aspectos económicos como de producción.

Actualmente existe un importante interés por considerar los efectos generados por la cosecha forestal sobre el medio ambiente y la manera de cómo disminuirlos, como también los costos y beneficios involucrados en esta actividad.

Muchas empresas forestales del país han elaborado en las últimas décadas planes de alta mecanización en la faena de cosecha forestal, con el objeto de enfrentar el aumento de los requerimientos de producción. Esto ha redundado en considerar los efectos de la mecanización en este tipo de faenas sobre el suelo, el cual representa uno de los recursos más importantes para la productividad del sitio forestal y el crecimiento de un bosque productivo. Una mala selección de la maquinaria y/o de un sistema de cosecha forestal, provocará impactos negativos sobre las propiedades físicas y químicas del suelo, conllevando una disminución de la productividad de los sitios forestales.

La selección de un tipo de sistema no es simple, los criterios involucrados presentan un carácter multivariante, la descripción y valoración de los mismos no se efectúa desde un único punto de vista, sino de múltiples perspectivas o criterios. Además, no todos los criterios involucrados en una evaluación poseen la misma significancia para quien decide. Por esta razón es necesario utilizar una metodología que permita asignar un peso o ponderación a cada criterio, de modo de asignar la importancia relativa a cada uno desde el punto de vista de un ente decisor, con el fin de aunar criterios para que la solución de la alternativa seleccionada sea realizada en base a los objetivos que se planteen la o las personas que toman la decisión.

Una solución a este problema es el uso de las técnicas de evaluación multicriterio, herramienta orientada a asistir en el proceso de toma de decisiones. Con estas técnicas es posible la selección de las máquinas y sistemas de cosecha más adecuados, de acuerdo a los criterios involucrados en la evaluación.

El presente estudio pretende elaborar, mediante la aplicación de la Técnica de Evaluación Multicriterio, una pauta que cuantifique el impacto sobre el suelo de las diferentes maquinarias de cosecha forestal (Skidder, Feller Buncher, y Forwarder) de acuerdo a criterios productivos, económicos y ambientales.

\section{METODOLOGÍA}

Mediante una recopilación bibliográfica se identificaron las variables del suelo que son afectadas por la cosecha mecanizada. Adicionalmente, se efectuó una revisión de los aspectos productivos y de protección al medioambiente de la maquinaria de cosecha más utilizada en Chile y en el extranjero. 
Una vez recopilada la información se obtuvieron los valores y las medidas restrictivas, las cuales se integraron mediante el uso de las técnicas de evaluación multicriterio (EMC).

Los temas que fueron integrados mediante el uso de las técnicas de evaluación multicriterio fueron los siguientes:

(a) Selección de los sistemas de cosecha más adecuados sobre la base de criterios productivos, económicos y ambientales.

(b) Selección del modelo de skidder, feller buncher, forwarder, harvester y trineumático más adecuado sobre la base de criterios productivos, económicos y ambientales.

(c) Selección de la serie de suelo forestal más apta para faenas de cosecha de acuerdo a criterios físicos y químicos.

\section{Evaluación Multicriterio}

Inicialmente se estableció la enumeración de $m$ puntos que representaron las posibles alternativas o elecciones alcanzables para el ente decisor $\left(A_{1}, A_{2}, \ldots, A_{i}, \ldots, A_{m}\right)$. Un tipo de alternativa considerada fue las máquinas ocupadas actualmente en faenas de cosecha en el país, como skidder, feller buncher y forwarder. Además, se requirió un conjunto de $\mathrm{n}$ puntos $\left(\mathrm{C}_{1}, \mathrm{C}_{2}, \ldots, \mathrm{C}_{\mathrm{j}}, \ldots, \mathrm{C}_{\mathrm{n}}\right)$ que representaron los atributos o criterios relevantes para el correspondiente problema de decisión. Una serie de criterios fue analizada para la maquinaria con sus respectivos sub criterios; productivos, económicos y ambientales.

Descritos los conceptos y dimensiones de la alternativa y los criterios, se estructuró adecuadamente la información que les relaciona y define. En primer lugar, un conjunto de $\mathrm{m}$ $x \mathrm{n}$ puntos $\left(\mathrm{R}_{11}, \ldots, \mathrm{R}_{\mathrm{ij}}, \ldots, \mathrm{R}_{\mathrm{mn}}\right)$, que representaron el resultado alcanzado por la alternativa 0 elección en cada uno de los atributos o criterios considerados. Para las diferentes maquinarias las matrices variaron su dimensión de acuerdo al número de alternativas y criterios que estos posean, para los skidders de $18 \times 9$, para los fellers de $12 \times 9$ y para los forwarders de $13 \times$ 11. Por otra parte se consideraron los pesos $\left(\mathrm{W}_{\mathrm{j}}\right)$ agrupados en el llamado vector de pesos, los cuales intentaron representar la estructura de preferencias del decisor (Barba-Romero y Pomerol, 1997).

\section{Cuantificación y Normalización de las Evaluaciones}

Las evaluaciones fueron las distintas características que la alternativa presentó frente a cada criterio, las que fue necesario cuantificar de la manera más precisa posible, ya que representaron a las alternativas en todo el proceso de análisis posterior. Para cada criterio la escala de medida de las evaluaciones estará muy determinada por su propia naturaleza.

Como en casi todos los métodos de EMC discretos, se precisa que las evaluaciones $r_{i j}$ de una alternativa concreta i, correspondiente a todos y cada uno de los criterios j, sea comparable en magnitud, unidad de medida, posición del cero, dispersión de medida, etc. Además, como cada escala de medida viene dada por su propia naturaleza (US $\$$, toneladas, $\mathrm{m}^{3} / \mathrm{h}$, etc.), no fue factible analizarlas de inmediato, por lo que fue necesario normalizar estos valores. En este 
estudio se aplicó el uso de escalas de medida, que poseen la particularidad de mantener la proporción entre los valores reales de las alternativas con respecto a cada criterio.

\section{Asignación de Pesos}

Como en todo problema de EMC es casi inevitable que algunos criterios tengan para el decisor más relevancia que otros, por razones personales éste puede considerar más importantes unos criterios que otros, dándole un peso relativo mayor o menor a los criterios.

Saaty (1977) introdujo un método multicriterio conocido por AHP (Analytic Hierarchy Process) o Proceso Analítico Jerárquico (PAJ) para la asignación de pesos a los factores.

Este procedimiento fue aplicado a este estudio con el objeto de asignar los pesos respectivos. Para ello se estableció una matriz cuadrada, en la cual el número de filas y columnas estuvo definido por el número de factores a ponderar. Así se estableció una matriz de comparación entre pares de factores, comparando la importancia de uno sobre cada uno de los demás $\left(\mathrm{C}_{\mathrm{ij}}\right)$, posteriormente se determinó el "egeinvector" principal, el cual estableció los pesos $\left(\mathrm{W}_{\mathrm{j}}\right)$ y el "egeinvalor" que proporcionó una medida cuantitativa de la consistencia de los juicios de valor entre pares de factores (Saaty, 1980).

Para valorar cada comparación por pares y rellenar las celdas de la matriz, se debió avanzar de columna en columna, de izquierda a derecha. En cada celda se determinó la importancia relativa de la variable de cada fila con relación a la variable de su columna correspondiente. La escala de medida establecida para la asignación de los juicios de valor es una escala de tipo continuo, que va de un valor mínimo de 1/9 hasta 9 , definida por Saaty (1980) como extremadamente menos importante (1/9), hasta extremadamente más importante (9), indicando el valor 1 igualdad de importancia entre pares de factores.

Para el cálculo de los pesos se utilizó el programa computacional IDRISI, en el cual, los pesos de los factores se visualizaron en la pantalla con su respectivo Coeficiente de Consistencia de la matriz, valor que indica la probabilidad de que los valores hayan sido asignados aleatoriamente. Valores inferiores a 0,10 indicaron buena consistencia. Cuando los valores excedían de 0,10 , la matriz de pesos fue evaluada nuevamente. Para la asignación de pesos para los diferentes criterios se contó con la participación de especialistas que ayudaron a asignar valores que representaron la importancia relativa que presentó cada criterio (Barredo, 1996).

Además se presentó la posible jerarquización de los criterios y la repercusión de ellos en los pesos que se les asignaron. Así una estructura jerárquica de criterios a dos niveles: un primer nivel de criterios "padres" y un segundo nivel de sub criterios "hijos" de aquellos. A los criterios padres se le asignó un valor de acuerdo a las preferencias del decisor y ese valor se repartió de manera diferente entre los criterios hijos (Barredo, 1996).

\section{Método de Ponderación Lineal} alternativa.

Para efectuar la ponderación lineal se calculó una puntuación global $\left(R_{i}\right)$ para cada 


$$
A_{i}: R_{i}=\sum_{j} W_{j}^{*} r_{i j}
$$

Se supone un problema de EMC con m alternativas y $\mathrm{n}$ criterios. La característica objetiva o la utilidad que, para el criterio j, el decisor estima tiene cada alternativa i, la recoge la evaluación $\mathrm{a}_{\mathrm{ij}}$ de la matriz de decisión.

Para la normalización de las evaluaciones $\mathrm{a}_{\mathrm{ij}}$ se realizó el procedimiento de escalas de valores de normalización que no alteran la proporcionalidad. Los nuevos $a_{i j}$ quedaron comprendidos entre 0 y 10, con mejor evaluación cuanto más cercanos a 10 se ubiquen, en el caso que criterios sean crecientes, o a maximizar, o decrecientes, o a minimizar.

Se normalizaron los pesos $\mathrm{W}_{\mathrm{i}}$ de forma que sumaran la unidad (basta dividir los pesos originales por la suma de todos ellos). A partir de esto se consideró que los $a_{i j}$ y los $W_{j}$ ya están normalizados según lo dicho.

Para cada alternativa i se calculó su puntuación global:

$$
\mathrm{R}_{\mathrm{i}}=\sum_{\mathrm{j}} \mathrm{W}_{\mathrm{j}} * \mathrm{r}_{\mathrm{ij}}(\mathrm{i}=1, \ldots, \mathrm{m})
$$

Donde: $W_{\mathrm{i}}$ : Pesos normalizados de cada criterio evaluador ${ }_{\mathrm{ij}}$ : Asignación de la puntuación individual de cada alternativa por su correspondiente criterio.

La alternativa a escoger fue aquella cuya puntuación global fue máxima. Si hubiera varias en esta situación, cualquiera de ellas sirve.

\section{RESULTADOS}

La maquinaria de cosecha forestal analizada involucró diversas marcas y modelos de skidder, feller buncher y forwarder, con el objeto de identificar aquella que presentara las características más relevantes de acuerdo a criterios productivos, económicos y ambientales.

De acuerdo a esto, se procedió a la evaluación de los pesos en relación a la experiencia de especialistas en esta área. Sobre esta base se determinaron los valores expresados en matrices de comparación por pares para los diferentes niveles de criterios evaluados, estas matrices se presentan en los siguientes cuadros. 
Cuadro $\mathrm{N}^{\circ} 1$

MATRIZ PARA ESTABLECER EL PESO DE LOS CRITERIOS EN LA SELECCIÓN DE LAS MAQUINARIAS MÁS ADECUADAS A TRAVÉS DEL PAJ CRITERIOS PADRES

\begin{tabular}{|c|c|c|c|c|}
\hline & CP1 & CP2 & CP3 & Pesos Xij \\
\hline CP1 & 1 & & & 0,6442 \\
\hline CP2 & $1 / 6$ & 1 & & 0,0852 \\
\hline CP3 & $1 / 3$ & 4 & 1 & 0,2706 \\
\hline
\end{tabular}

Cc: 0,05

El Cuadro $\mathrm{N}^{\circ} 1$ de criterios padres es similar para todos los tipos de maquinaria evaluados, por lo tanto se efectuó una evaluación general de ésta. Aquí se observa que los criterios productivos presentan el mayor peso $(64,42)$, de modo que son los que poseen mayor importancia para el ente decisor, seguido de los criterios ambientales $(27,06)$ y los económicos $(8,52)$ en nivel de importancia. Se determinó que los criterios productivos (CP1) son los más importantes, debido al rol que cumplen en el cumplimiento de las metas planificadas para satisfacer las exigencias de demanda que poseen las empresas forestales.

Siguiendo en importancia están los criterios ambientales (CP3), ya que estos están relacionados con el efecto de la compactación superficial por presión sobre el suelo y sub superficial (peso bruto), el cual produce la alteración de las características físicas del suelo, como perdida de porosidad, aumento de la densidad aparente y disminución de la capacidad de infiltración de agua y aire, efectos que disminuyen la productividad de los sitios forestales.

Además se observa que el coeficiente de consistencia es menor a 0,10, por lo tanto se puede decir que los juicios de valor asignados son consistentes.

Cuadro $\mathrm{N}^{\circ} 2$

MATRIZ PARA ESTABLECER EL PESO DE LOS CRITERIOS EN LA SELECCIÓN DEL SKIDDER MÁS ADECUADO A TRAVÉS DEL PAJ SUBCRITERIOS PRODUCTIVOS

\begin{tabular}{|c|c|c|c|c|c|c|}
\hline & Scp1 & Scp2 & Scp3 & Scp4 & Scp5 & Pesos Xij \\
\hline Scp1 & 1 & & & & & 0,2112 \\
\hline Scp2 & $1 / 2$ & 1 & & & & 0,1535 \\
\hline Scp3 & $1 / 2$ & $1 / 2$ & 1 & & & 0,1118 \\
\hline Scp4 & 3 & 4 & 3 & 1 & & 0,4587 \\
\hline Scp5 & $1 / 3$ & $1 / 3$ & $1 / 2$ & $1 / 5$ & 1 & 0,0648 \\
\hline
\end{tabular}

Cc: 0,04

Dentro de los sub criterios productivos, el rendimiento (Scp4) es el de mayor importancia $(45,87)$, seguido por los criterios de potencia (Scp1 $(21,12))$, fuerza de tracción (Scp2 $(15,35))$, capacidad de carga (Scp3 $(11,18)$ ) y velocidad máxima de desplazamiento (Scp5 $(6.48)$ ) a la cual se le adjudicó el menor peso de todos los criterios involucrados.

Criterios de potencia y fuerza de tracción están relacionados con la fuerza de arrastre 
disponible y la capacidad de las máquinas de confrontar situaciones adversas, como lo es superar la resistencia al rodado y de pendiente.

Se observa que el coeficiente de consistencia es menor a 0,10 , por lo tanto se puede decir que los juicios de valor asignados son consistentes.

\section{Cuadro $\mathrm{N}^{\circ} 3$}

MATRIZ PARA ESTABLECER EL PESO DE LOS CRITERIOS EN LA SELECCIÓN DEL FELLER BUNCHER MÁS ADECUADO A TRAVÉS DEL PAJ. SUBCRITERIOS PRODUCTIVOS

\begin{tabular}{|c|c|c|c|c|c|c|}
\hline & Scp1 & Scp2 & Scp3 & Scp4 & Scp5 & Pesos Xij \\
\hline Scp1 & 1 & & & & & 0,2257 \\
\hline Scp2 & $1 / 2$ & 1 & & & & 0,1135 \\
\hline Scp3 & 3 & 3 & 1 & & & 0,4274 \\
\hline Scp4 & $1 / 3$ & 2 & $1 / 4$ & 1 & & 0,1505 \\
\hline Scp5 & $1 / 2$ & $1 / 2$ & $1 / 3$ & $1 / 3$ & 1 & 0,0829 \\
\hline
\end{tabular}

Cc: 0,08

El rendimiento (Scp3) y la potencia (Scp1), al igual que en los Skidders, siguen siendo los sub criterios de mayor importancia dentro de los criterios productivos, con pesos de 42,.74 y 22,57, respectivamente, seguidos por diámetro máximo de corte (Scp4), con un peso de 15,05, capacidad de carga Scp2, con 11,35, y por último, la pendiente máxima de trabajo (Scp5) con 8,29 . La capacidad de carga tiene una ponderación muy baja.

Finalmente, el sub criterio de pendiente máxima es el que posee el menor peso debido a que la pendiente de trabajo de las alternativas evaluadas no difiere mucho una de otra.

El coeficiente de consistencia es 0,08 , muy cercano a 0,10 , pero todavía menor, por lo que se puede decir que los juicios de valor asignados son consistentes.

\section{Cuadro $\mathrm{N}^{\circ} 4$}

MATRIZ PARA ESTABLECER EL PESO DE LOS CRITERIOS EN LA SELECCIÓN DEL FORWARDER MÁS ADECUADO A TRAVÉS DEL PAJ SUBCRITERIOS PRODUCTIVOS

\begin{tabular}{|l|c|c|c|c|c|c|c|c|}
\hline & Scp1 & Scp2 & Scp3 & Scp4 & Scp5 & Scp6 & Scp7 & Pesos Xij \\
\hline Scp1 & 1 & & & & & & & 0,2197 \\
\hline Scp2 & $1 / 2$ & 1 & & & & & & 0,1349 \\
\hline Scp3 & 3 & 6 & 1 & & & & & 0,3741 \\
\hline Scp4 & $1 / 5$ & $1 / 5$ & $1 / 9$ & 1 & & & & 0,0278 \\
\hline Scp5 & $1 / 6$ & $1 / 2$ & $1 / 4$ & 3 & 1 & & & 0,0617 \\
\hline Scp6 & $1 / 3$ & $1 / 2$ & $1 / 2$ & 5 & 4 & 1 & & 0,1360 \\
\hline Scp7 & $1 / 3$ & $1 / 4$ & $1 / 5$ & 2 & $1 / 2$ & $1 / 4$ & 1 & 0,0458 \\
\hline
\end{tabular}

Cc: 0,07

Se presenta un mayor número de sub criterios productivos, pero de similar forma que en los casos anteriores, el rendimiento (Scp3) y la potencia (Scp1) son los que presentan la mayor 
ponderación con un peso de 37,41 y 21,97, respectivamente. Los siguen en importancia los sub criterios pendiente máxima de trabajo (Scp6) con 13,60, capacidad de carga (Scp2), con 13,49, capacidad de la garra (Scp5) con 6,17, alcance máximo (Scp7), con 4,58, y por último el sub criterio velocidad máxima (Scp4) con la más baja ponderación $(2,78)$.

La capacidad de la garra y el alcance máximo no poseen una ponderación tan alta, debido a que la diferencia entre las alternativas evaluadas no es tan significante. Algo que es notorio, respecto del caso anterior del feller buncher, es el mayor peso asignado a la capacidad de carga, esto se debe a que para este tipo de máquina es fundamental este tipo de criterio, ya que esa es su labor, la de transportar la madera, lo cual esta directamente relacionado con su rendimiento.

El coeficiente de consistencia es menor a 0,10 , por lo que se concluye que los juicios de valor aplicados en este caso son consistentes.

Respecto de los sub criterios ambientales para los diferentes tipos de maquinaria, en los cuadros siguientes se entregan los resultados de su análisis.

\section{Cuadro $\mathrm{N}^{\circ} 5$}

MATRIZ PARA ESTABLECER EL PESO DE LOS CRITERIOS EN LA SELECCIÓN DEL SKIDDER MÁS ADECUADO A TRAVÉS DEL PAJ SUB CRITERIOS AMBIENTALES

\begin{tabular}{|c|c|c|c|c|}
\hline & Sca1 & Sca2 & Sca3 & Pesos Xij \\
\hline Sca1 & 1 & & & 0.1429 \\
\hline Sca2 & 2 & 1 & & 0.2857 \\
\hline Sca3 & 4 & 2 & 1 & 0.5714 \\
\hline
\end{tabular}

Cc: 0,00

Se puede observar que el sub criterio ambiental de presión sobre el suelo de los neumáticos traseros (Sca3), fue al que se le asignó mayor importancia relativa $(57,14)$, ya que es aquí donde se concentra todo el peso de la carga arrastrada. Seguido en nivel de importancia se encuentra la presión de las ruedas delanteras (Sca2) con 28,57, y por último el peso bruto de la máquina con el menor valor de importancia $(14,29)$, debido a la mayor compactación sobre la superficie por la presión de los neumáticos, que aquella producida en los horizontes más profundos y que esta relacionada con el peso de la maquinaria.

El coeficiente de consistencia es 0,00 , menor a 0,10 , por lo tanto los juicios de valor asignados son consistentes.

Cuadro $\mathrm{N}^{\circ} 6$

MATRIZ PARA ESTABLECER EL PESO DE LOS CRITERIOS EN LA SELECCIÓN DEL FELLER BUNCHER MÁS ADECUADO A TRAVÉS DEL PAJ. SUB CRITERIOS AMBIENTALES

\begin{tabular}{|c|c|c|c|c|}
\hline & Sca1 & Sca2 & Sca3 & Pesos Xij \\
\hline Sca1 & 1 & & & 0,1311 \\
\hline Sca2 & 4 & 1 & & 0,6608 \\
\hline Sca3 & 2 & $1 / 4$ & 1 & 0,2081 \\
\hline
\end{tabular}

Cc: 0,05 
A diferencia del caso de los skidders, la presión sobre el suelo de las ruedas delanteras (Sca2), es el sub criterio ambiental que se le asigna el mayor grado de importancia $(66,08)$, debido a que es aquí donde se concentra el mayor esfuerzo que realiza este tipo de máquinas, desplazando al segundo lugar al sub criterio ambiental de presión sobre las ruedas traseras (Sca3) cono 20,81, y por último, y similar al caso de los skidders, se le dio la menor ponderación al peso bruto de la máquina (Sca1) con 13,11. Esto se debe, al igual que en el caso de los skidders, a que el efecto que se produce en la superficie del suelo es más relevante, directamente relacionado con la presión de los rodados, que aquel que producen en horizontes más profundos, directamente relacionados con el peso de las máquinas.

El coeficiente de consistencia es 0,05 , menor a 0,10 , por lo que los juicios de valor asignados son consistentes.

\section{Cuadro $\mathrm{N}^{\circ} 7$}

MATRIZ PARA ESTABLECER EL PESO DE LOS CRITERIOS EN LA SELECCIÓN DEL FORWARDER MÁS ADECUADO A TRAVÉS DEL PAJ SUB CRITERIOS AMBIENTALES

\begin{tabular}{|c|c|c|c|c|}
\hline & Sca1 & Sca2 & Sca3 & Pesos Xij \\
\hline Sca1 & 1 & & & 0,1047 \\
\hline Sca2 & 3 & 1 & & 0,2583 \\
\hline Sca3 & 5 & 3 & 1 & 0,6370 \\
\hline
\end{tabular}

Cc: 0,03

En el análisis de los sub criterios ambientales se observa que el sub criterio presión sobre el suelo de las ruedas traseras (Sca3), es el que presenta la mayor ponderación $(63,70)$ de parte del ente decisor, considerando a este como el más importante dentro de los criterios ambientales, ya que, al igual que en los skidders, es acá donde se concentra la mayor cantidad de la presión sobre el suelo. Lo siguen en importancia los sub criterios presión sobre el suelo de las ruedas delanteras (Sca2) con 25,83, y por último el sub criterio peso bruto de la máquina (Sca1), con la más baja ponderación $(10,47)$.

Esto se basa en que la alteración provocada en la superficie del suelo es mucho más importante en la regeneración de un bosque, ya que provoca efectos de compactación, afectando más los horizontes explorados por las raíces; reduciendo la permeabilidad y el intercambio gaseoso, alteraciones directamente relacionada con la presión de los rodados, que los horizontes más bajos, lo que está relacionado con el peso del equipo.

El coeficiente de consistencia es menor a 0,10 , por lo que los juicios de valor aplicados en esta situación son consistentes.

Corresponde ahora el análisis de criterios hijos de algunos sub criterios evaluados, resultados que se presentan en los cuadros siguientes. 


\section{Cuadro $\mathrm{N}^{\circ} 8$ \\ MATRIZ PARA ESTABLECER EL PESO DE LOS CRITERIOS EN LA SELECCIÓN DEL FELLER BUNCHER MÁS ADECUADO A TRAVÉS DEL PAJ SUB SUBCRITERIOS PRODUCTIVOS}

\begin{tabular}{|c|c|c|c|c|c|}
\hline & Sscp3.1 & Sscp3.2 & Sscp3.3 & Sscp3.4 & Pesos Xij \\
\hline Sscp3.1 & 1 & & & & 0,5545 \\
\hline Sscp3.2 & $1 / 2$ & 1 & & & 0,2999 \\
\hline Sscp3.3 & $1 / 6$ & $1 / 3$ & 1 & & 0,1000 \\
\hline Sscp3.4 & $1 / 8$ & $1 / 6$ & $1 / 2$ & 1 & 0,0556 \\
\hline
\end{tabular}

Cc: 0,01

Estos sub sub criterios corresponden a la disminución del rendimiento debido al aumento de la pendiente. Aquí se ve que la asignación de importancia relativa de estos criterios va en forma descendente de acuerdo al aumento de la pendiente, ya que existe el supuesto de que las máquinas son empleadas generalmente en pendientes suaves, que no superan el $20 \%$.

Sin embargo, en el caso particular de que la evaluación se hubiera realizado pensando en un terreno con pendientes más fuertes, la asignación de los pesos hubiera sido diferente, realzando la importancia de aquel rango de pendiente que el terreno en estudio posea. Así se puede variar la asignación de los juicios de valor de acuerdo a los objetivos que se requiere cumplir.

Mediante el establecimiento del Proceso Analítico Jerárquico (PAJ) a todas la matrices de las maquinarias evaluadas, se obtuvieron los pesos $\left(\mathrm{W}_{\mathrm{f}}\right)$ correspondientes a los criterios. Asimismo, el coeficiente de consistencia indica que la asignación de los juicios de valor es consistente. Con esto se obtuvo la matriz de decisión para los sistemas de cosecha forestal, en la cual los datos aparecen presentados en su escala natural de medida (Cuadros $\mathrm{N}^{\circ} \mathrm{s}$ 9, 11 y 13). Además se determinó para cada criterio, si es creciente o a maximizar o decreciente o a minimizar, el tipo de escala de medida utilizado para el proceso de normalización de los datos, y los valores de los parámetros de las escalas de medida.

Se normalizaron los valores de cada criterio, con el objeto de llevar a la unidad la sumatoria de los pesos, luego realizar la aplicación de la sumatoria ponderada lineal para obtener la puntuación global y por ende la ordenación de las alternativas evaluadas, como se muestra en los Cuadros $N^{\circ}$ s 10, 12 y14).

La ordenación final de la matriz del Cuadro $\mathrm{N}^{\circ} 9$ muestra que el skidder $\mathrm{N}^{\circ} 8$, Caterpillar, modelo Grapple 528 de neumáticos, es el que obtiene mayor puntuación global, el que presenta mejores características de acuerdo a los criterios ocupados para su evaluación.

Este modelo de skidder no presenta las mejores características en todos los criterios evaluados, pero si en una mayor cantidad que el resto de las alternativas, que de acuerdo a la importancia relativa de los criterios en la cual es superior, le confieren una mayor puntuación, lo que le permite ser considerado aquel modelo con mejores condiciones para desarrollar el trabajo requerido. 
Al comparar este modelo que obtuvo la primera puntuación global, y por ende la primera ordenación (skidder $\mathrm{N}^{\circ} 8$ ), con el modelo que obtuvo la segunda puntuación global, el skidder Timberjack cable 460 de neumáticos, se ve que la primera alternativa es superior en casi la totalidad de los criterios productivos evaluados: Respecto del costo de inversión y del peso de la máquina, es superado por la segunda alternativa (skidder $\left.\mathrm{N}^{\circ} 15\right)$. Pero como en este caso, los criterios productivos tienen mayor ponderación que aquellos ambientales y económicos, la alternativa $\mathrm{N}^{\circ} 8$ tuvo finalmente mayor puntuación global que el modelo $\mathrm{N}^{\circ} 15$, seleccionándolo en primera prioridad.

\section{Cuadro $\mathrm{N}^{\circ} 9$}

\section{MATRIZ DE DECISIÓN PARA LOS DISTINTOS MODELOS Y MARCAS DE SKIDDERS}

\begin{tabular}{|c|c|c|c|c|c|c|c|c|c|c|}
\hline \multirow[b]{4}{*}{ Alternativas } & Criterios & \multicolumn{5}{|c|}{ CP1 } & CP2 & \multicolumn{3}{|c|}{ CP3 } \\
\hline & \multirow{2}{*}{\begin{tabular}{|l} 
Peso $=100$ \\
Subcriterios
\end{tabular}} & \multicolumn{5}{|c|}{64,42} & 8,52 & \multicolumn{3}{|c|}{27,06} \\
\hline & & Scp1 & Scp2 & Scp3 & Scp4 & Scp5 & Sce1 & Sca1 & Sca2 & Sca3 \\
\hline & Peso $=100$ & 13,61 & 9,89 & 7,20 & 29,55 & 4,17 & 8,52 & 3,87 & 7,73 & 15,46 \\
\hline 1 & & 95 & 110 & 3.325 & 99 & 21,5 & 175.000 & 7.700 & 50,7 & 55,3 \\
\hline 2 & & 95 & 110 & 3.325 & 105 & 21,5 & 195.000 & 8.766 & 50,7 & 55,3 \\
\hline 3 & & 140 & 133 & 4.900 & 87 & 23 & 140.000 & 13.331 & 50,7 & 55,3 \\
\hline 4 & & 140 & 133 & 4.900 & 92 & 23 & 185.000 & 12.497 & 50,7 & 55,3 \\
\hline 5 & & 160 & 193 & 5.600 & 61 & 23,5 & 89.000 & 13.857 & 42,5 & 46,2 \\
\hline 6 & & 160 & 193 & 5.600 & 74 & 23,5 & 105.000 & 13.558 & 42,5 & 46,2 \\
\hline 7 & & 175 & 210 & 6.125 & 82 & 28 & 150.000 & 14.039 & 46 & 49,9 \\
\hline 8 & & 175 & 210 & 6.125 & 90 & 28 & 165.000 & 14.719 & 46 & 49,9 \\
\hline 9 & & 116 & 230 & 4.060 & 59 & 10,3 & 135.000 & 12.909 & 57 & 57 \\
\hline 10 & & 116 & 230 & 4.060 & 68 & 10,3 & 148.000 & 14.031 & 62 & 62 \\
\hline 11 & & 142 & 300 & 4.970 & 65 & 10,9 & 165.000 & 17.677 & 63 & 63 \\
\hline 12 & & 142 & 300 & 4.970 & 74 & 10,9 & 180.000 & 18.801 & 66 & 66 \\
\hline 13 & & 115 & 155 & 4.025 & 60 & 24,8 & 102.000 & 9.933 & 50,7 & 55,3 \\
\hline 14 & & 165 & 205 & 5.775 & 86 & 29,2 & 170.000 & 12.973 & 46 & 49,9 \\
\hline 15 & & 174 & 146 & 6.090 & 88 & 29 & 153.000 & 11.282 & 46 & 49,9 \\
\hline 16 & & 174 & 146 & 6.090 & 94 & 32 & 190.000 & 14.512 & 46 & 49,9 \\
\hline 17 & & 116 & 120 & 4.060 & 55 & 24 & 132.000 & 8.387 & 50,7 & 55,3 \\
\hline 18 & & 116 & 120 & 4.060 & 64 & 26 & 148.000 & 10.061 & 50,7 & 55,3 \\
\hline
\end{tabular}




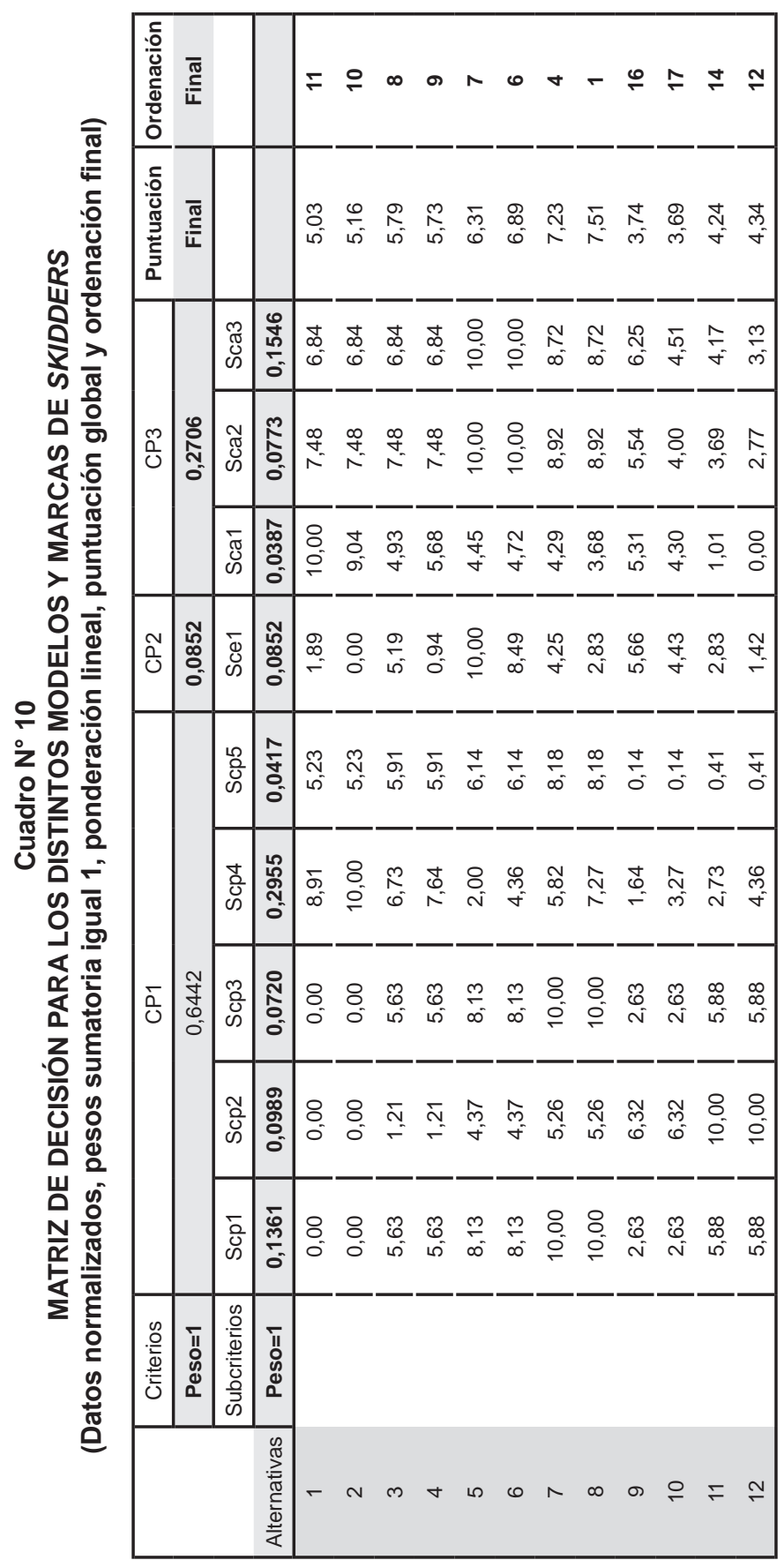




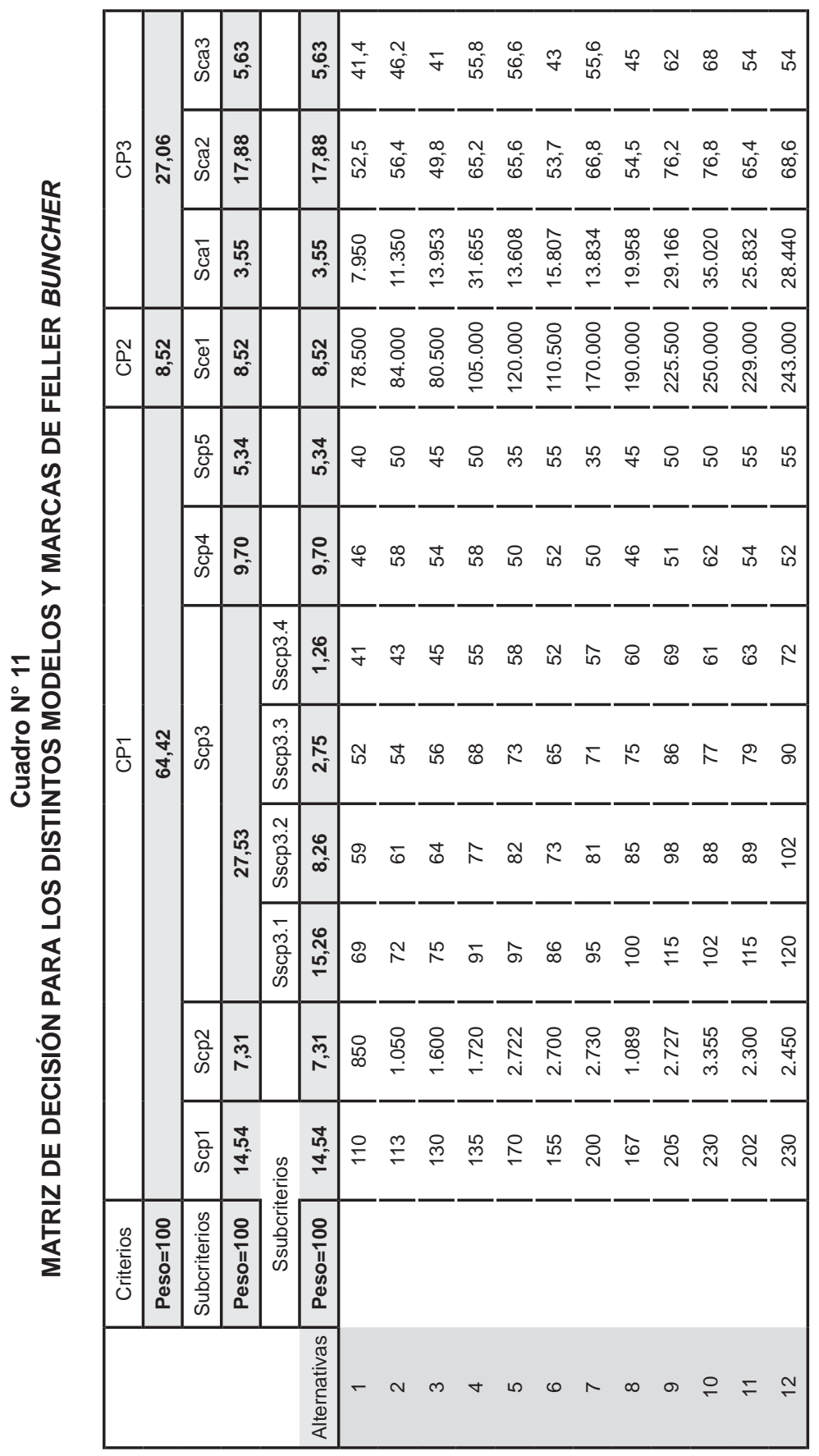




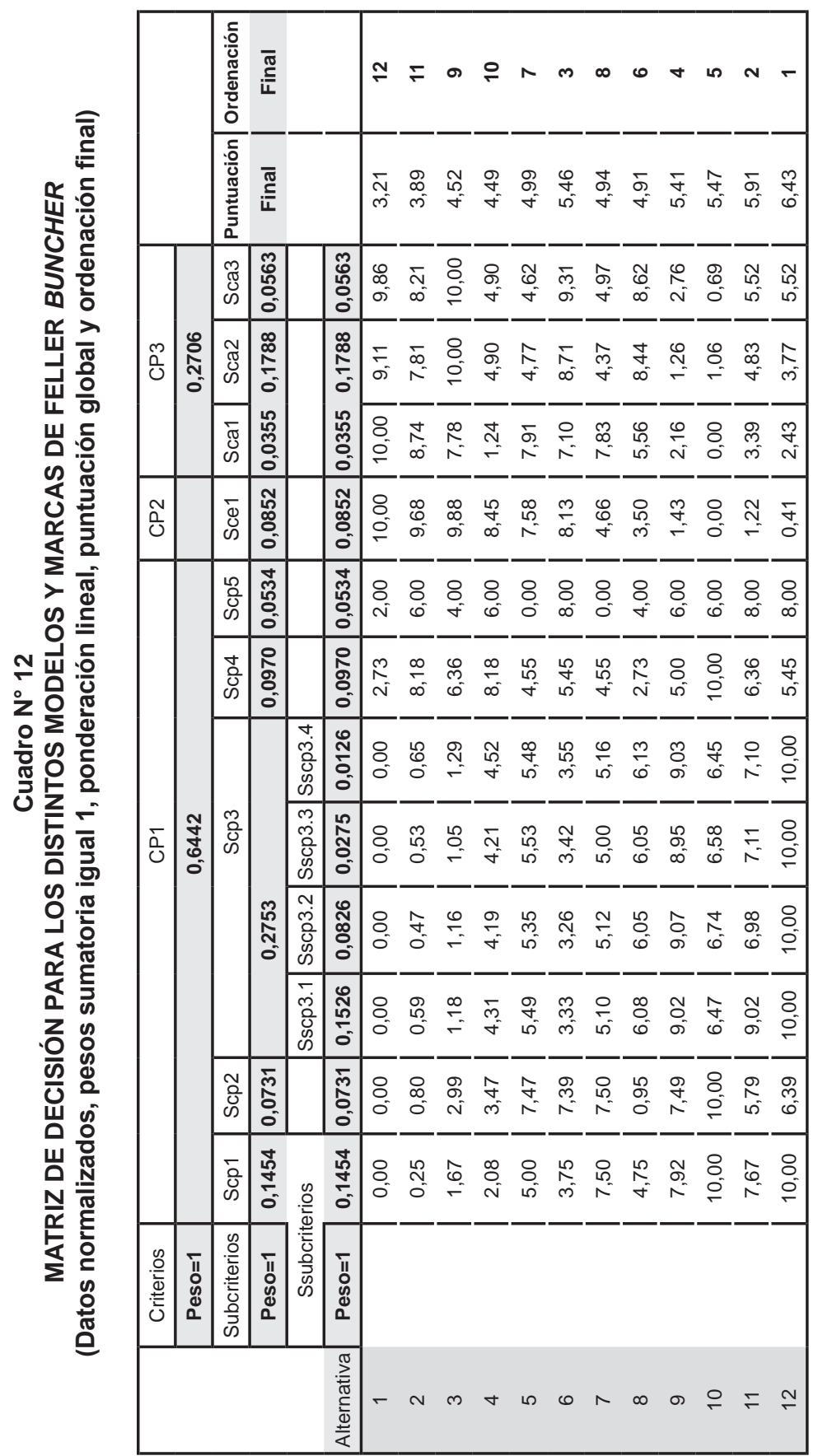




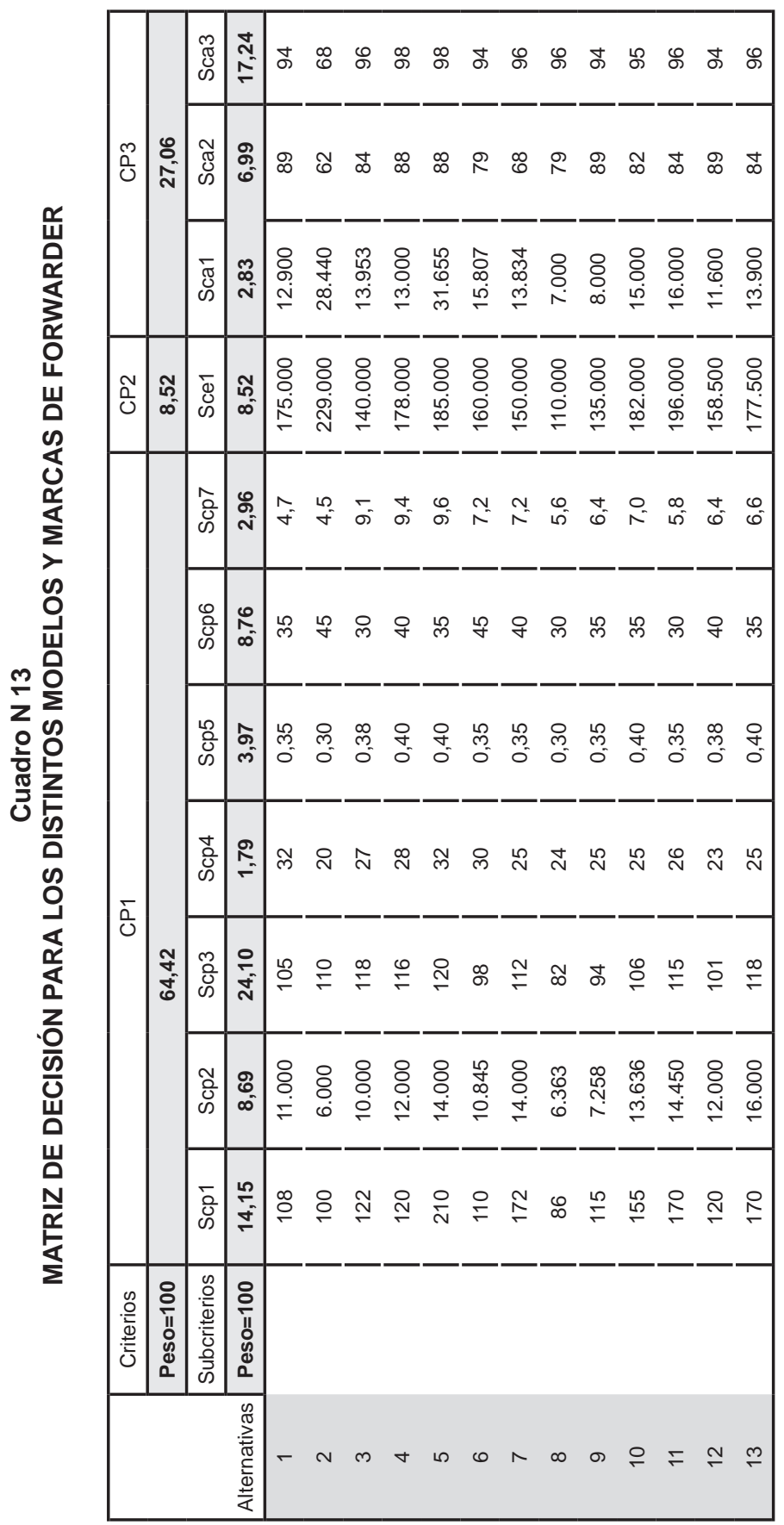




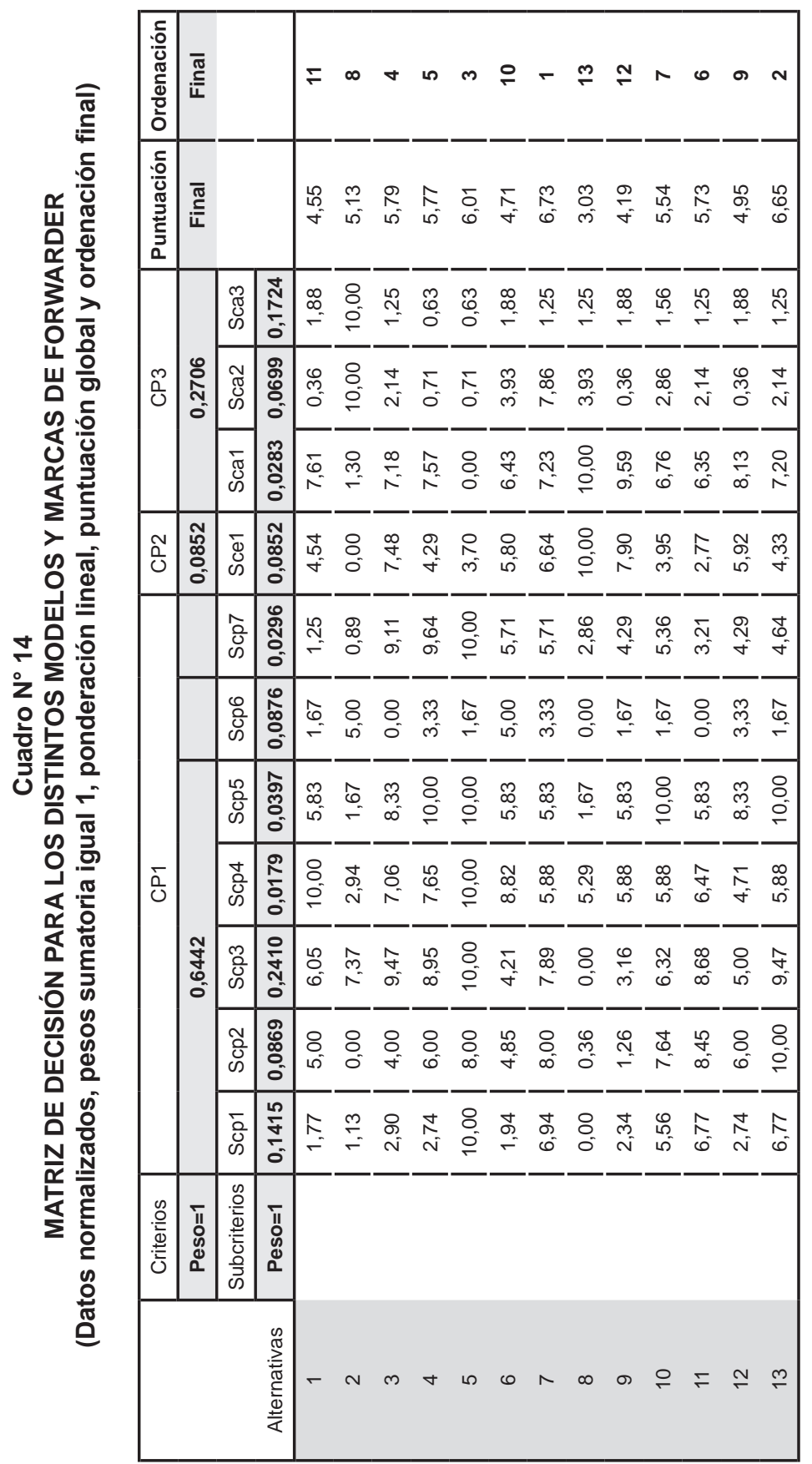


Se observa en el Cuadro $\mathrm{N}^{\circ} 11$ que la alternativa $\mathrm{N}^{\circ} 12$, el Feller Buncher Timberjack 2628 de orugas, es la alternativa que obtuvo la mayor puntuación global, o sea, de acuerdo a los criterios evaluados ésta es la primera preferencia en el caso de tener que seleccionar una de estas máquinas para una faena de cosecha forestal, ya que, globalmente, es la alternativa que presenta mejores condiciones de productividad, económicas y ambientales.

La alternativa $\mathrm{N}^{\circ}$ 2, o sea, el Feller Buncher Timberjack 2618 de orugas, tiene mejores características que la alternativa seleccionada con la primera prioridad en los criterios de diámetro máximo de corte, en el costo de inversión y en el peso total de la máquina, pero es sobrepasado en el resto de los criterios, y como estos poseen una mayor ponderación para sus pesos le otorgan a esa alternativa una mayor puntuación global.

El análisis de los resultados de la ponderación lineal de la matriz del Cuadro $\mathrm{N}^{\circ} 11$ muestra que la alternativa $\mathrm{N}^{\circ} 7$, fue la que obtuvo la mayor puntuación global, y por lo tanto la primera preferencia para el ente decisor. Esta alternativa corresponde al Forwarder Timberjack $1210 \mathrm{~B}$ de neumáticos. Este modelo correspondería a aquel que cumple de mejor forma con las restricciones que impuso el decisor.

Al comparar las evaluaciones de este modelo con la primera preferencia, con un caso extremo como es aquella alternativa que obtuvo la más baja preferencia de acuerdo a los criterios involucrados en la evaluación (alternativa N 8, Forwarder Fabtek FT244 de neumáticos), se observa que esta última es mejor en los criterios de costo de inversión y de peso bruto de la máquina, pero superado claramente en el resto de los criterios. Esto demuestra que ninguna de las alternativas aquí evaluadas es dependiente de otra, o sea, en por lo menos uno de los criterios involucrados en la evaluación, es superior al resto de las alternativas, lo que le permite seguir siendo involucrado en la posterior evaluación.

Esta ordenación final depende mucho de los objetivos que posea el ente decisor, ya que esta ordenación se realiza a base de la mayor o menor puntuación durante la asignación de los juicios de valor, lo que le va a otorgar el grado de importancia a un criterio en particular, que hará destacar por sobre las otras alternativas a aquella que posea mejores características en ese determinado criterio.

\section{CONCLUSIONES}

Las variables del suelo más afectadas por la cosecha mecanizada fueron la densidad aparente, porosidad, drenaje, y contenido de materia orgánica.

Los modelos de sistemas de cosecha forestal evaluados que presentaron mejores características de trabajo y un menor daño al suelo, fueron: Grapple Skidder Caterpillar 528, Feller Buncher Timberjack 2628, y Forwarder Timberjack 1210B. 


\section{REFERENCIAS}

Barba-Romero, S. y J.C. Pomerol. 1997. Decisiones multicriterio: fundamentos teóricos y utilización práctica. Alcalá de Henáres (Madrid): Servicio de Publicaciones de la Universidad de Alcalá. 420 p.

Barredo, J., 1996. Sistemas de Información Geográfica y Evaluación Multicriterio en la Ordenación del Territorio. Madrid, España: Editorial Ra-Ma. Primera edición. 263p.

Saaty, T., 1977. AScaling Method for Priorities in Hierarquical Structures. Journal of Mathematical Psychology. No 15. pp. 234-281.

Saaty, T., 1980. The Analytic Hierarchy Process. Mc Graw Hill. New York. 
